\title{
ИНФОРМАЦИОННАЯ СИСТЕМА ТЕРМИНАЛЬНОГО ТИПА ОПЕРАТИВНОГО ДОСТУПА К РЕСУРСАМ ЦИФРОВОГО ПРОИЗВОДСТВА
}

\author{
М. В. Паринов, А. Н. Юров, И. А. Еремин \\ Воронежский государственный технический университет
}

Поступила в редакцию 26.02.2018 г.

\begin{abstract}
Аннотация. В статье рассмотрена реализация аппаратно-программного решения в виде информационного терминального клиента для системы управления индивидуальным жизненным циклом (ИЖЦ) изделия ЛОЦМАН. Особенностью разработки является возможность использования продукта персоналом с невысокой квалификацией вследствие создания упрощенного клиентского модуля с простым эргономичным интерфейсом; продукт предназначен для использования в условиях цеха и имеет соответствующее исполнение. Ключевые слова: управление жизненным циклом изделия, терминал, цифровое производство, клиент-серверная архитектура, доступ к информационным ресурсам.

Annotation. Hardware-software solution for product life management system that made like terminal client for PLM LOCMAN is presented in this article. The main solutions feature is ability to using terminal by not professional staff in manufacturing facilities. The client has simple ergonomic interface and protected construction.

Keywords: for product life management, terminal, digital industry, client-server design, informational access.
\end{abstract}

В сложившихся мировых условиях возникает острая необходимость разработки отечественных продуктов с целью замены ими аналогов западного производства. В том числе данная проблема актуальна для программных продуктов, обеспечивающих функционирование цифрового производства. В настоящее время цифровое производство обычно строится на базе системы управления жизненным циклом изделия. Примером отечественного продукта подобного класса является ЛОЦМАН от компании АСКОН.

Использование отечественных систем управления ИЖЦ решает не только задачу импортозамещения, но и обеспечивает ряд дополнительных преимуществ. К ним относятся: глубокая поддержка отечественных стандартов (например, ЕСКД, ЕСТД, ЕСПД и т. д.), улучшенная адаптация и кастомизация под нужды отечественного производства.

Система ЛОЦМАН является сложным распределенным клиент-серверным продук-

() Паринов М. В., Юров А. Н., Еремин И. А., 2018 том. В стандартную поставку входят клиентские и серверные программы, обеспечивающие автоматизацию мест инженерных работников. Стандартное клиентское место требует наличия типового персонального компьютера и высокую подготовку работающего на нем специалиста.

В производственных условиях данные требования часто являются невыполнимыми. Например, в условиях цеха использования компьютера в незащищенном исполнении обычно недопустимо вследствие несоответствующих условий функционирования (пыль, прочие загрязнения, вибрация, влага и так далее). Однако наиболее острым является вопрос подготовки специалистов. Условия современного цифрового производства требуют обеспечить доступ к инженерной документации для работников любого уровня. Применение промежуточных звеньев, когда данные передаются от одного специалиста к другому, существенно снижают эффективность и надежность производства. При этом необходимо учитывать, что разрешение до- 


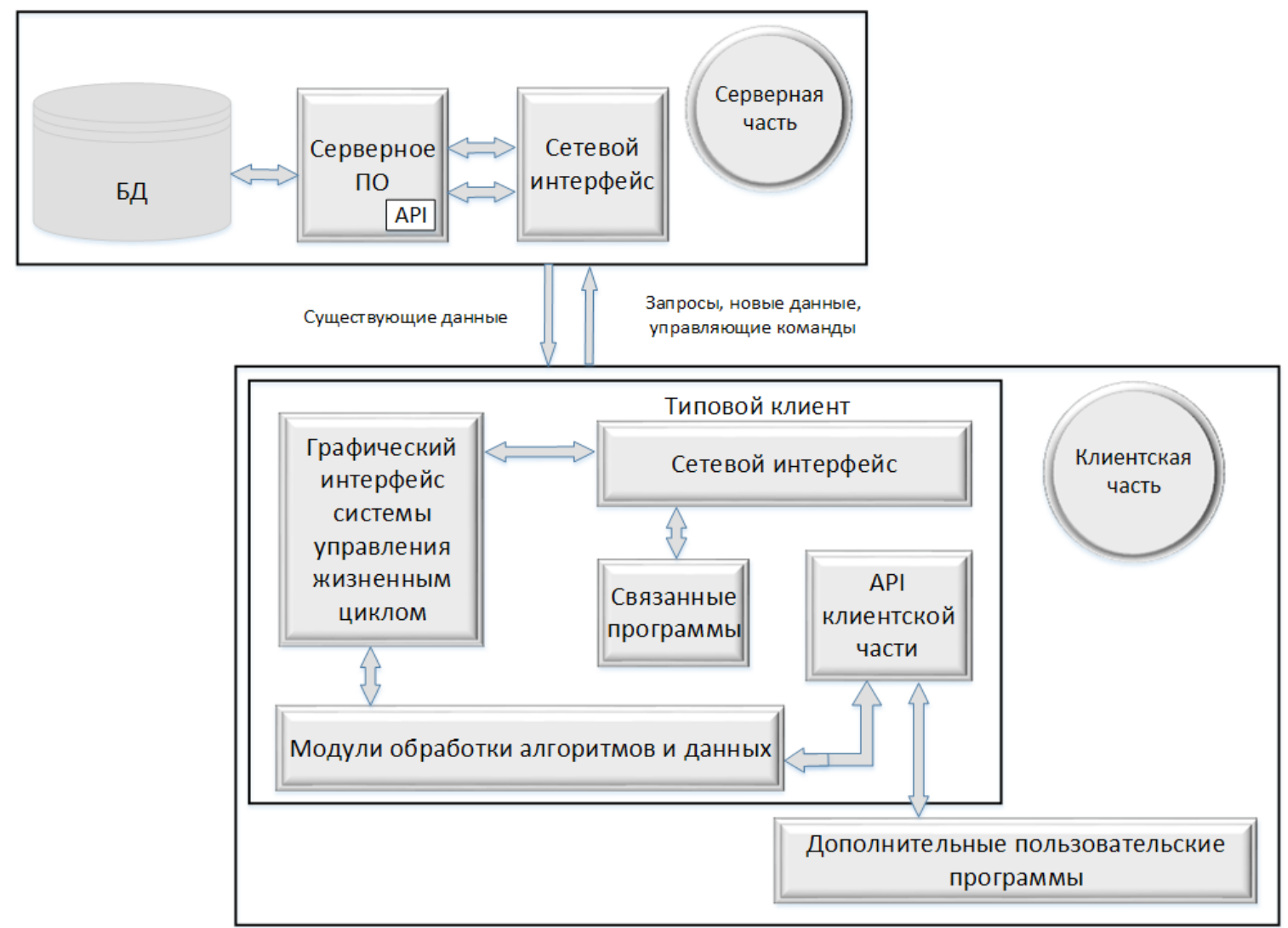

Рис. 1. Типовая схема базовой системы ЛОЦМАН

ступа к инженерной информации для некомпетентных работников не приведет к положительным результатам, а также может стать причиной ошибок и аварийных ситуаций.

Отсюда следует задача: обеспечить возможность взаимодействия работников различного уровня с системой управления жизненным циклом ЛОЦМАН. Задача решается методом адаптирования аппаратных средств к производственным условиям и преобразования информации в пригодную для эффективного использования требуемыми работниками форму.

Рассмотрим типовую схему информационных потоков в системе ЛОЦМАН - рис. 1. Все информационные ресурсы цифрового производства хранятся в базе данных, являющей неотъемлемым элементом серверной части системы. Для работы с базой данных используется специализированное серверное программное обеспечение, в том числе включающее программный интерфейс серверной части ЛОЦМАН. Реализация связи с сетевыми ресурсами, входящими в состав системы, представлена соответствующими модулями.
Посредством сетевых модулей создается связь с клиентской частью системы. Протоколы обмена данными между стандартным клиентом и серверным ПО являются закрытой информацией. Для связи сторонних программных продуктов с серверной частью предусмотрен программный интерфейс.

Типовой клиент содержит схожий модуль сетевого обмена. Для доступа и манипулирования данными предусмотрен многофункциональный графический интерфейс. Графический интерфейс взаимодействует с функциональными элементами программной части, которые реализуют ядро клиентской части. Клиент ЛОЦМАН собственный программный интерфейс, к которому могут быть подключены пользовательские подпрограммы. Также клиентская часть допускает подключение дополнительных программных продуктов, входящих в состав информационной системы цифрового производства.

Проблема адаптации типовой клиентской части для решения поставленной задачи заключается в высокой сложности стандартных интерфейсных средств и излишней (для 
Информачионная система терминального типа оперативного доступа к ресурсам циифрового ...

работника невысокой/непрофильной квалификации) функциональности. Второстепенной проблемой является большое количество значков и надписей стандартного интерфейса, основная часть из которых имеет малые размеры.

Поэтому первый необходимый шаг в решении поставленной задачи - определение функциональности программного продукта для доступа к инженерным данным. Анализ реальных производственных задач показывает, что в условиях цеха наибольшую актуальность имеет получение справочной информации об определенных объектах, которые можно найти по ключевым параметрам (например, идентификаторам, названиям, связям и так далее). Данные должны быть представлены в максимально простой форме.

Обращение с устройством должно быть интуитивно понятным, не требовать специальных навыков. Интерфейс должен быть рассчитан на реализацию эргономичного защищенного исполнения. В частности, в цеховых условиях основной упор целесообразно делать не на стандартные средства (мышь + клавиатура), а сенсорный экран с клавиатурой и трекболом в качестве дополнительных (не часто используемых) средств управления.

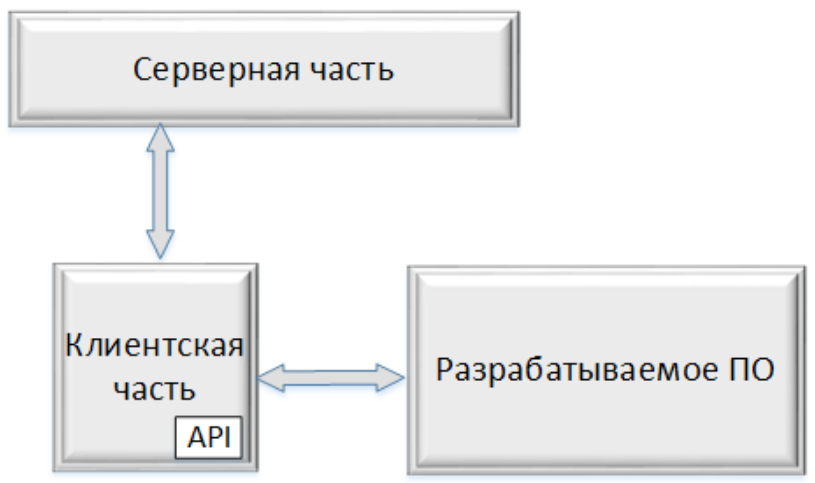

Рис. 2. Схема подключения программь

Для реализации указанных требований необходимо создать специализированное программное средство, основной задачей которого является преобразование, представление информации и интерфейсное взаимодействие с пользователем. Как следует из рисунка 1 , клиентская часть имеет программный интерфейс, к которому может быть подключена данная программа - рис. 2.
Однако рассматриваемое решение имеет значительный недостаток: необходимость установки на конечном клиентском устройстве типового клиента, а также снижение скорости доступа к информационным ресурсам, повышение задержек, вероятности возникновения ошибок. Поэтому оптимальным решением будет подключение разрабатываемой программы непосредственно через программный интерфейс серверной части.

Окончательное решение схематично показано на рис. 3. Клиентская часть, выполненная в виде специальной программы, содержит сетевой модуль, через который она связывается с программным интерфейсом серверной части системы. При этом наличие элементов стандартного клиента ЛОЦМАН не требуется.

Графический интерфейс (рис. 4) выполнен в стиле информационного терминала и оптимизирован для работы с сенсорным экраном. Клавиатура и указательное устройство (трекбол) используются только для ввода символьной информации и для повышения эргономичности управления. Особенности интерфейса обеспечивают работу с программой работников с минимальной квалификацией; отсутствие маленьких значков и редко используемых функций значительно повышают удобство применения в условиях цеха.

Интерфейс взаимодействует с модулем обработки запросов и фильтрации. Он обеспечивает основной функционал клиентской части. Реализует запросы к серверным компонентам, их предварительную и окончательную фильтрацию, а также получение данных. Добавление данных в базу не предусмотрено спецификой продукта.

Получаемая информация подлежит предварительной обработки для оптимальной формы вывода на экран. Для большинства форматов предварительным контейнером является pdf файл. Модуль визуализации обеспечивает корректное масштабирование и отображение как двумерных, так и трехмерных объектов, запрашиваемых из системы.

Аппаратная часть клиентского модуля построена на базе обычного персонального компьютера, помещенного в защищенный корпус 


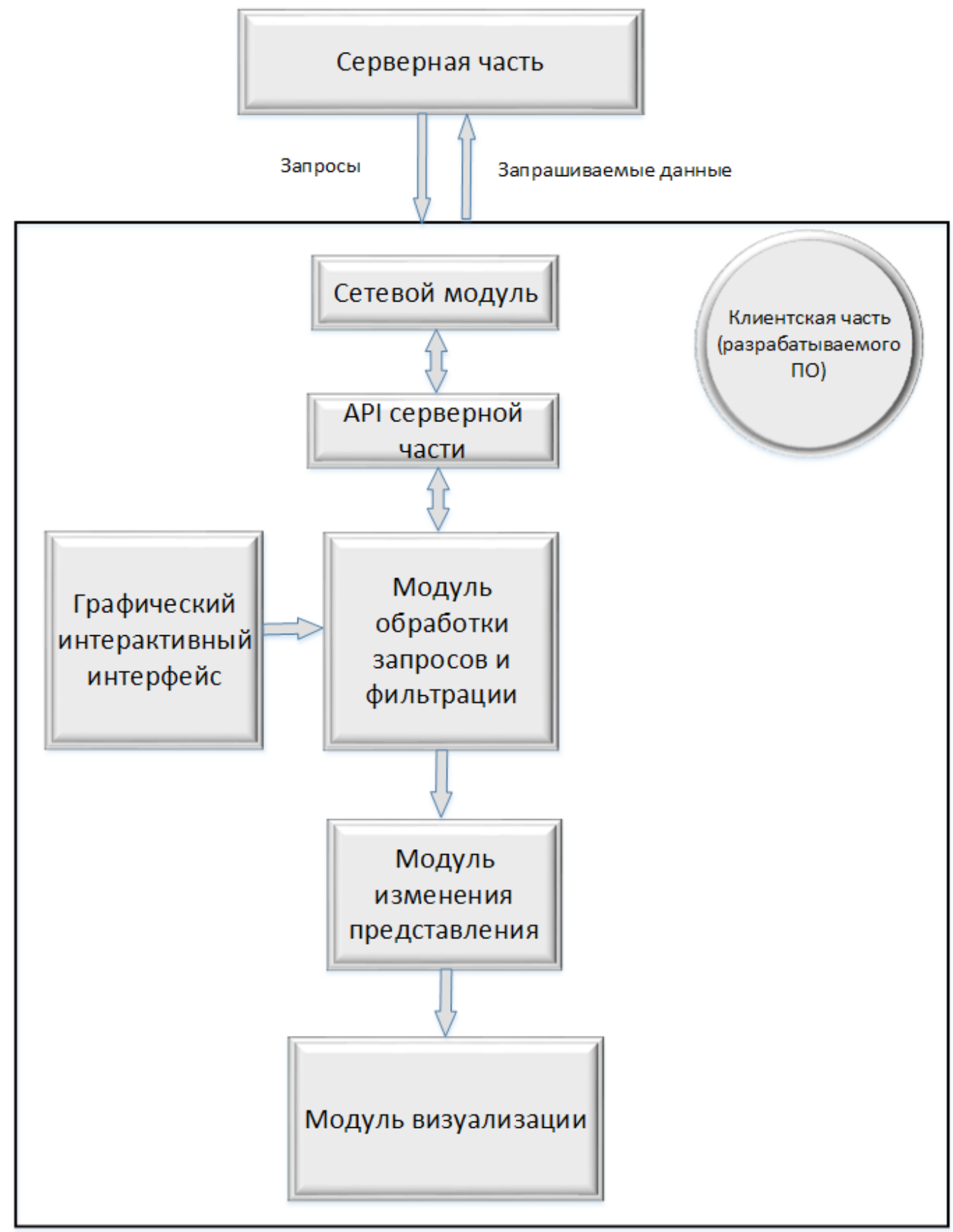

Рис. 3. Схема разработанной программы

в виде информационного терминала - рис. 5. минального клиента ИЖЦ ЛОЦМАН. РасИсполнение корпуса и его защита выбираются согласно требованиям предприятия, эксплуатирующего устройство. Опционально с целью повышения эргономичности терминал может быть оснащен принтером. В большинстве случаев конструкция аппаратной части не несет значимой новизны, и его можно считать обычным персональным компьютером.

Исходя из наиболее актуальных задач типового машиностроительного производства нами реализован перечень функций для терсмотрим основные из них:

- поддержка многопользовательского режима работы с сервером ЛОЦМАН и наличие отдельного информационного интерфейс по авторизации пользователя;

- возможность проверять и вносить инженерные запросы;

- наличие навигации по найденным объектам;

- реализация подготовки критериальных списков по типам документов; 
Информачионная система терминального типа оперативного доступа к ресурсам цииррового ...

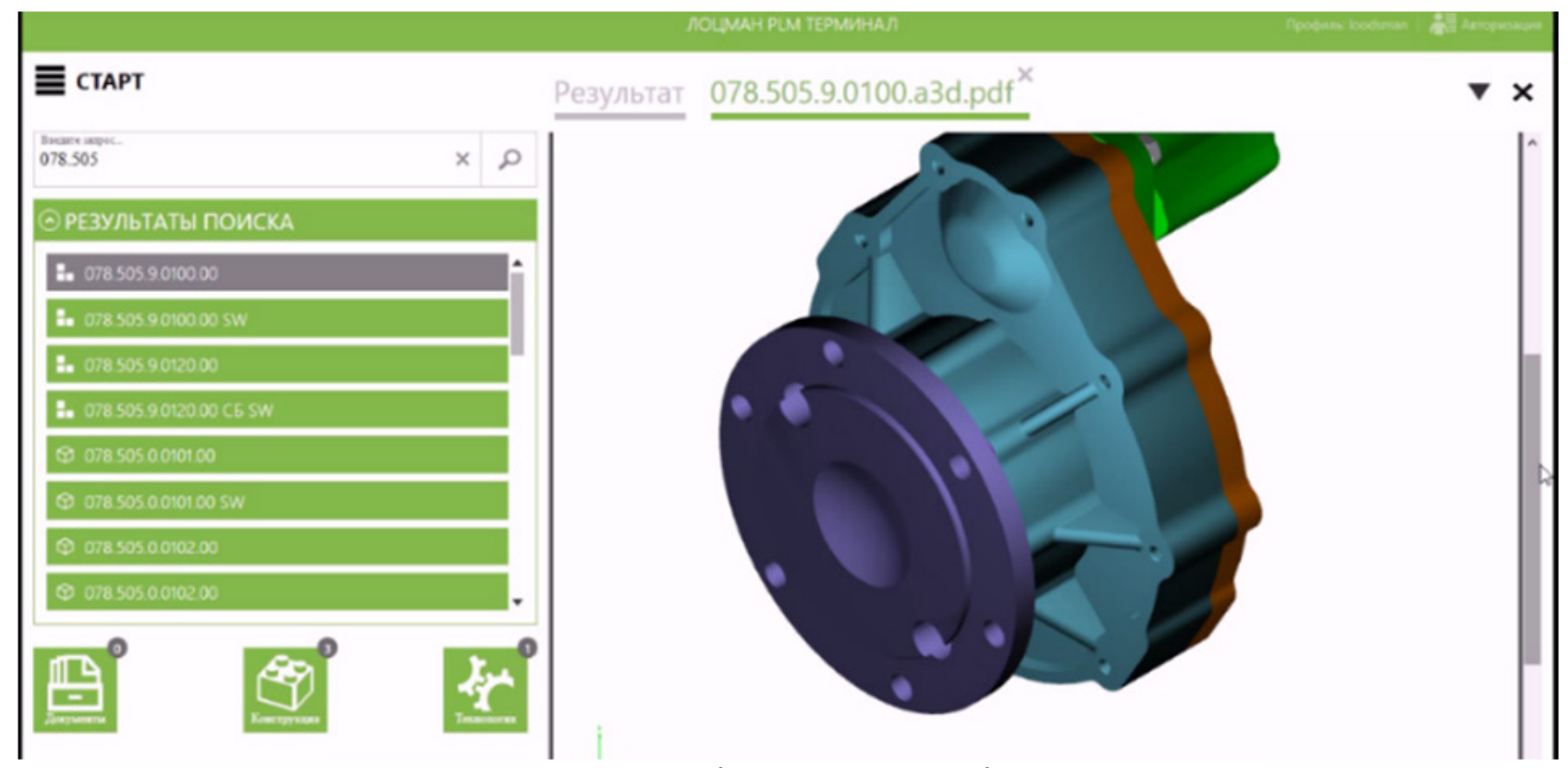

Рис. 4. Графический интерфейс

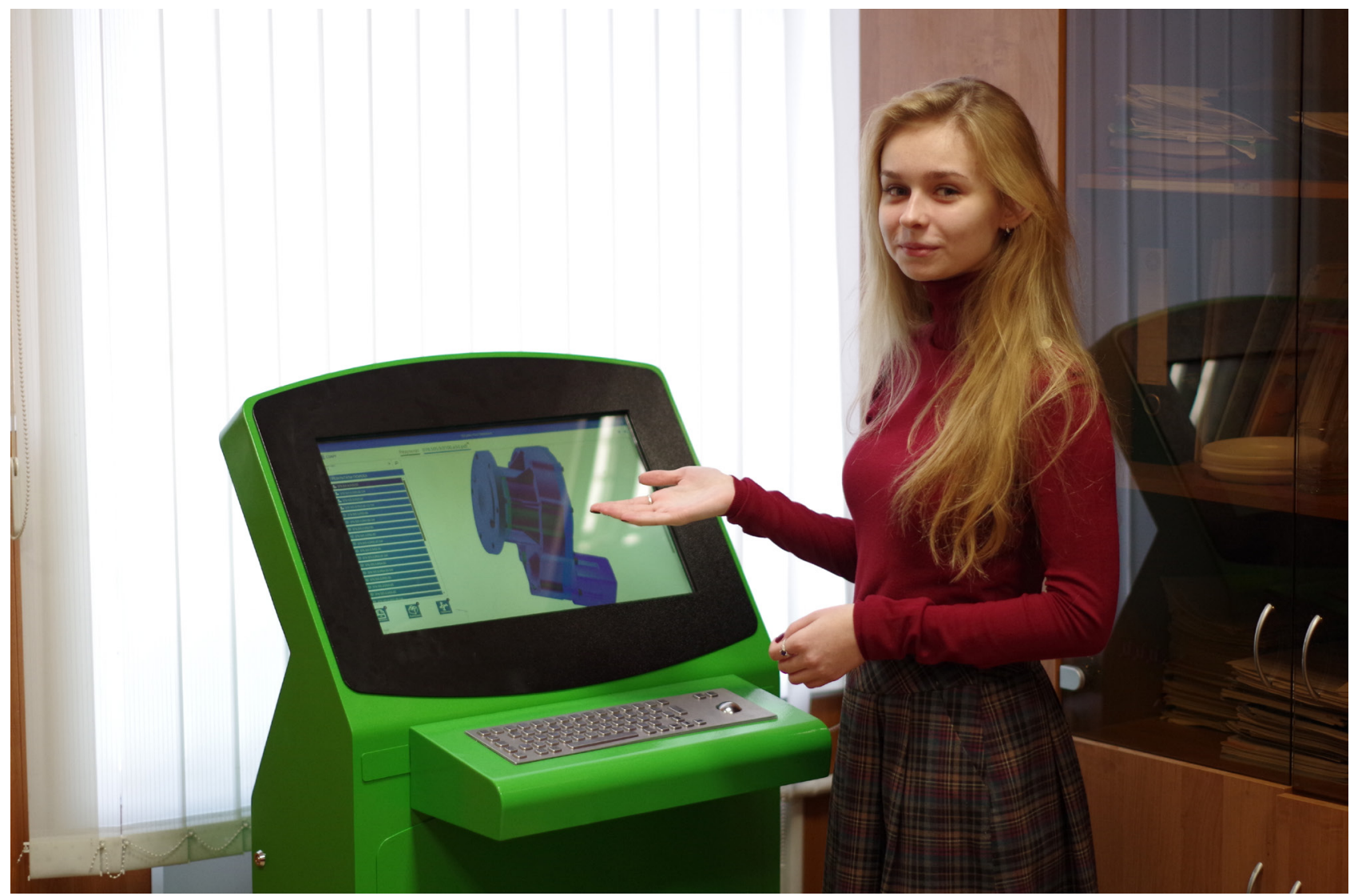

Рис. 5. Аппаратное исполнение решения

- реализация структурированного набора инженерных данных по запросу на локальном хранилище данных;

- реализация по отображению перечня документов согласно указанным ранее критериям;
- визуализация сборочных единиц в отдельном окне приложения;

- визуализация твердотельных моделей в отдельном окне приложения;

- отображение чертежных документов и спецификаций в отдельном окне приложения; 
- подготовлена визуализация прочих инженерных данных подобным образом, как предложено в работе;

- реализация поддержки в проектном решении графических форматов данных, а также имеет возможность обратиться к форматам данных текстовых процессоров.

Одной из ключевых особенностей разработанной программы является использование технологии разработки интерактивного интерфейса Windows Presentation Foundation (WPF). Применение данной технологии позволило создать эргономичный эстетичный интерфейс, обеспечивающий автоматическое масштабирование под различное разрешение экрана. Данная опция очень важна при установке терминального клиента на устройства с различными дисплеями. Предложенная разработка обеспечивает корректное отображение данных при любом разрешении, начиная от 1280 х 1024 точек.

Для организации связи с сервером используется технология DCOM. Выбор технологии объясняется требованиями текущей версии серверных компонентов системы ЛОЦМАН. Текущая реализация DCOM соединения требует размещения информационного терминала в едином домене с сервером ЛОЦМАН. В последующих разработках планируется использование дополнительных методов сетевых соединений для расширения гибкости предложенного решения.

Доступ к данным, хранимым в системе ЛОЦМАН, осуществляется с помощью типового программного интерфейса (API). Для реализации терминального программного клиента не требуется установки стандартного клиентского приложения ЛОЦМАН на устройство. Необходимым является только комплект библиотек, обеспечивающих создание сетевого соединения. Все функции, обеспечивающие реализацию перечисленных задач, реализуются посредством AРI серверных компонентов ЛОЦМАН.

Основные задачи терминального клиента в текущей версии реализуют следующие функции:

- обработку данных, полученных от серверной базы данных ЛОЦМАН;
- конвертацию и предварительную обработку накопленных ранее записей в базе данных ЛОЦМАН;

- хранение файлов с инженерными данными как в преобразованном виде, так и исходном формате, доступном для последующего редактирования;

- формирование списков по заданным фильтрам и критериям;

- создание иерархической древовидной структуры данных всего перечня данных;

- реализация многофункционального поиска по заданным параметрам, которые гибко выбираются пользователем системы;

- экспорт данных в формат PDF;

- отображение данных в формате PDF, форматы текстовых процессоров и ряд графических форматов;

- авторизация и защита от несанкционированного доступа для просмотра, изменения и копирования данных;

- разграничение уровня пользователей по доступным для них функциям системы:

- администраторы (полный доступ);

- пользователи (просмотр данных);

- создание пользовательских фильтров для поиска и анализа полученных данных из внешней базы Лоцман;

- сетевой доступ к файлам, полученных в ходе работы с серверной частью системы;

- оптимизация временных и не востребованных в работе файлов как локально, так и на сетевом хранилище.

Bсе перечисленные выше функции направлены для получения информации согласно предусмотренного уровня доступа. Информация о производственных объектах представляется в удобном интерактивном режиме с целью обучения и информирования цеховых работников. Ряд перечисленных функций являются вспомогательными и являются необходимыми для реализации основных задач.

В результате реализации представленной разработки нами был получен аппаратно-программный комплекс, обеспечивающий получение информации в условиях производства из ИЖЦ системы ЛОЦМАН согласно запросам пользователей. Использование раз- 
Информачионная система терминального типа оперативного доступа к ресурсам циифрового ...

работки не требует специальной подготовки пользователей, а защищенное исполнение позволяет разместить продукт непосредственно в цеху.

\section{СПИСОК ЛИТЕРАТУРЫ}

1. Рыжков, В. А. Разработка системы визуализации разнородных данных цифрового макета изделия / В. А. Рыжков // Известия Са-

Паринов М. В. - канд. техн. наук, доцент, доцент кафедры Компьютерных интеллектуальных технологий проектирования Воронежского государственного технического университета.

E-mail: parmax@mail.ru

Юров А. Н. - канд. техн. наук, доцент, доцент кафедры Компьютерных интеллектуальных технологий проектирования Воронежского государственного технического университета. E-mail: yurovalex@mail.ru

Еремин А. А. - старший преподаватель кафедры Компьютерных интеллектуальных технологий проектирования Воронежского государственного технического университета. марского научного центра Российской академии наук. - 2016. - Т. 18, № 4-3. - С. 634-637.

2. Собенина О. В. Разработка поискового модуля автономного клиента системы Teamcenter / O. В. Собенина, В. А. Рыжков, А. А. Пак // Информатика: проблемы, методология, технологии: труды Межд. конф. Воронеж: ФГБОУ ВО «Воронежский государственный технический университет». 2017. - C. 331-336.

Parinov M. V. - Ph. D of Technical Sciences, associate professor, Computer intellectual technology of design department, Voronezh state technical university.

E-mail:parmax@mail.ru

Yurov A. N. - Ph. D of Technical Sciences, associate professor, Computer intellectual technology of design department, Voronezh state technical university.

E-mail:yurovalex@mail.ru

Eremin I. A. - Computer intellectual technology of design department, Voronezh state technical university. 